\title{
HCC incidence and recurrence after DAAs: new insights
}

\author{
Necati Örmeci \\ Department of Gastroenterology, Ankara University Medical School, Ankara 06590, Turkey. \\ Correspondence to: Prof. Necati Örmeci, Department of Gastroenterology, Ankara University Medical School, Ankara 06590, Turkey. \\ E-mail: normeci@yahoo.com
}

How to cite this article: Örmeci N. HCC incidence and recurrence after DAAs: new insights. Hepatoma Res 2019;5:11.

http://dx.doi.org/10.20517/2394-5079.2019.14

Received: 27 Feb 2019 First Decision: 4 Mar 2019 Revised: 13 Mar 2019 Accepted: 14 Mar 2019 Published: 18 Apr 2019

Science Editor: Guang-Wen Cao Copy Editor: Cai-Hong Wang Production Editor: Huan-Liang Wu

Hepatit $\mathrm{C}$ virus (HCV) infection is a global health and economic problem in the world. It is the cause of chronic active hepatitis, liver cirrhosis, hepatic decompensation, hepatocellular carcinoma (HCC) and extrahepatic manifestations. Before 2013, standard care of HCV infection was the combination of Pegylated Interferon (PEG IFN) and Ribavirin. Sustained Virologic Response (SVR) rate of this combination was approximately $50 \%$ after 48 weeks of therapy. One fifth of patients who had this combination had to stop treatment because of severe side effects. Adding of Telaprevir or Boceprevir (first generation of protease inhibitors) to PEG IFN and Ribavirin therapy for 24 or 48 weeks result in a SVR rate around 70\%. However, because of severe adverse events, these combinations are not recommended.

$\mathrm{HCV}$ infection is the second most common cause of death in man, and incidence of HCC varies between $2 \%-8 \%$ in cirrhotic patients in a year. In a number of studies, it was shown that IFN-based treatments reduce the complication of advanced liver diseases such as decompensation, or HCC, liver related or all causes of mortality ${ }^{[1-6]}$. Although occurrence of SVR after the treatment of IFN-based or IFN free therapies is associated with a decrease of HCC occurrence, it does not eliminate the disease entirely, and HCC occurs annually in a rate of $0.4 \%-2 \%$ in advanced liver disease ${ }^{[5]}$.

Ninety HCV related cirrhotic patients were 1:1 randomized to receive IFN alfa thrice in a week for 12-24 weeks and as controls. SVR rate was $16 \%$ and HCC occurred in 19 patients who were followed up for 2-7 years. Two out of 19 patients had SVR while the remaining 17 did not have SVR $(P=0.002)^{[6]}$.

Direct-Acting Antivirals (DAAs) is a revolution for the treatment of HCV infection with a more than 95\% of SVR rates. Long-term results of treatment with DAAs on liver parenchymal disorders, hepatic

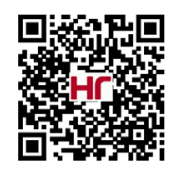


decompensation and occurrence or recurrence rates after radical treatment of HCC are not fully understood.

Recently several studies reported unexpectedly high occurrence or recurrence rates of HCC after DAAs treatment for HCV and curative treatment for $\mathrm{HCC}^{[1,7,8]}$.

In a retrospective study, 344 patients with liver cirrhosis were treated with DAAs. 285 of those patients did not have a history of HCC, but 59 patients did. SVR rate was $91 \%$. Six months after follow-up, 26 patients were shown to have HCC (7.6\%). HCC occurred in 9 out of 285 (3.2\%) patients without a history of HCC, while recurrence of HCC developed in $17(28.8 \%)$ patients with a previous history of HCC. Besides, disease free survival was short as a median of 376 days in this group. They postulated that SVR after the treatment with DAAs was not associated with the reduced risk of HCC occurrence. However this study had several gaps such as historical control was used instead of normal control group, absence of information in detail about surgical resection of HCC for 59 patients with a history of HCC preceding the treatment of DAAs, and radiological control was performed in 6 months rather than 3 months. There were different HCC treatment modalities such as ablation, resection, and percutaneous ethanol injection. In addition, there were differences in terms of confounding factors between the treatment groups, and DAAs were started as a median of 376 days after the curative treatment of HCC. Normally HCC recurrence occurs at a rate of $20 \%$ after curative treatment of HCC. Unexpected increased rate of HCC recurrence (28.8\%) may be a missed diagnosis of remnant HCC in several cases ${ }^{[1]}$.

In another similar study, 58 patients with HCV-related early HCC who achieved complete response after curative HCC treatment [resection $n$ : 20; ablation $n$ : 32; Trans Arterial Chemo Embolization (TACE) $n: 6$ ] were treated with DAAs after 11.2 months. SVR rate was 97.5\%. Median follow-up time was 5.7 (0.4-14.6) months. Sixteen patients (27.6\%) had unexpected recurrence of HCC in a median of 3.5 (1.1-8) months. It is difficult to definitely estimate early recurrence of HCC due to small sample size of study, high clinical, biological, and epidemiological heterogeneity of early $\mathrm{HCC}^{[7]}$.

In a meta-analysis which included 30 observational studies, 31,538 patients were treated for IFN-based regimen. SVR occurred in 10,853 patients (34.4\%). Overall HCC occurrence was 5.5\%. A hundred forty-five out of 9,185 patients (1.5\%) with SVR developed HCC while 990 out of 16,312 patients (6.2\%) had HCC. No matter if the patient had varying degrees of fibrosis or advanced liver disease, SVR was associated with 54\% reduction in all causes of mortality, histologic improvement, risk for progression of liver disease, liver related mortality, and $\mathrm{HCC}^{[8]}$.

Ravi et al. ${ }^{[9]}$ reported 6 out of $66 \mathrm{HCV}$ patients with HCC (9.1\%) after the treatment of DAAs during 6 months follow-up. Cardoso et al.$^{[10]}$ reported that HCC occurrence rate was $7.4 \%$ in 54 patients with cirrhotic HCV treated with DAAs after 12 months of follow-up. Yang et al. ${ }^{[1]}$ compared the 81 patients who had liver transplantation in terms of HCC occurrence. The patients receiving DAAs in pre-liver transplantation showed $27.8 \%$ of HCC while those who did not take DAAs demonstrated 9.5\% (6/63) of HCC. Among 17,487 patients who were treated with DAAs, 624 of them had HCC. 142 patients with HCC received liver transplantation. 482 patients still had HCC. Overall SVR was found 91\% in non-HCC, $74 \%$ in HCC, $94 \%$ in transplanted due to HCC. It was concluded that the patients who had transplantation due to HCV and HCC can successfully be treated with DAAs with high SVR rates ${ }^{[12]}$.

In a large retrospective cohort study comparing 2,400 chronic hepatitis $\mathrm{C}$ patients treated with IFN and 490 untreated patients, all patients had a liver biopsy, and they were followed up for a mean of 4.3 years. HCC occurred in 89 treated and 59 untreated patients. It was found that both F2 and F3 patients had less HCC in treated group compared to untreated group ( $P=0.0128$ for $F 2$; $P=0.0011$ for F3 patients). Predictive factors for less HCC occurrence were SVR, normal Alanine Aminotransferase (ALT) levels, and less than two times 
of the upper normal limits for aspartate aminotransferase (AST) level ${ }^{[13]}$. In another retrospective study, 463 patients with HCV cirrhosis were treated with the combination of PEG interferon and Ribavirin. Three hundred of 463 patients had SVR (64.8\%). Development of HCC was seen in 3 and 9 patients with SVR and non-SVR, respectively. It was found that SVR group had less HCC (1\%) compared to non-SVR group (5.5\%) $(P=0.005)$ after 36.1 months follow-up ${ }^{[14]}$.

Innes et al. ${ }^{[15]}$ reported that 857 patients with $\mathrm{HCV}$ infection were treated with IFN-based or IFN free regimens to compare the occurrence of HCC in both groups. Patients were followed up for 2.4 years, and 46 patients out of 857 had HCC. Incidence of HCC occurrence was two-fold high [(2.53 vs. 1.26 per 100 person years) $P=0.21$ in patients who took IFN free regimen. However, those patients were more thrombocytopenic, were at the higher Child Pugh stages, had more treatment experiences and were older than the patients who took IFN-based regimens. When confounding factors were corrected, there was no difference between two treatment regimens in terms of HCC occurrence. Adjusted (HR: 1.15, 95\%CI: 0.49$2.71 ; P=0.744)$.

Similarly, Telep et al. ${ }^{[16]}$ presented US administrative claims data which contained 4,887 patients who were curatively treated for HCC and HCV infection. Those patients were treated with IFN-based or IFN free treatment regimens. The latter patients were followed up for 182 days while the former were followed up 349 days. The patients in the IFN free treatment regimen had cirrhosis more frequently (95.7\% vs. $88.2 \%)$, more liver necrosis (34.8\% vs. 9.8\%), more portal hypertension (58\% vs. $35.3 \%$ ) and were older than the patients who took IFN-based treatment regimen. Statistical difference could not be shown after adjusting the confounding factors (HR: 0.97; 95\%CI: 0.49-1.92) between two treatment regimen groups in terms of HCC occurrence after following three, six and twelve months periods.

In a large retrospective cohort study, Group A ( $n=3534$, PEG IFN treated group) and Group B $(n=834$, Sofosbuvir and Simeprevir or Sofosbuvir and Ledipasvir treatment group), Group C ( $n=8468)$ untreated group were compared for occurrence of HCC in patients with liver cirrhosis. There were not statistical differences for basic characteristics among the groups. Mean follow-up time was 2,719.2 days for IFN-treated persons and 396.4 days for DAAs-treated persons. It was found that there was no association between HCC occurrence rates and DAAs treatment compared to IFN treatment ${ }^{[17]}$.

In an Italian multi-centric study, 328 patients with HCV related early HCC followed up for the recurrence of HCC. Median time for the recurrence of HCC was 31 months (26-38) in the group of active hepatitis, 72 months in the group of SVR by Interferon free therapies, 82.3 months in group of SVR by Interferon based therapies. There were statistical differences between active hepatitis and entire SVR groups. However there was not a difference between Interferon free or Interferon based treatment groups. In the multivariate analysis, serum bilirubin, creatinine and alpha-feto protein (AFP) levels were found to be an independent predictor for recurrence of $\mathrm{HCC}^{[18]}$.

In a well-designed prospective large cohort study, 143 consecutive HCV infected patients who had complete response after curative treatment of HCC with stage Barcelona Clinic Liver Cancer (BCLC) 0/A were treated with DAAs. Those patients were followed up for a mean of 9.1 (3-19) months. SVR rate was $96 \%$. The 6-, 12 - and 18-month HCC recurrence rates in the whole cohort were $12 \%, 26.6 \%$ and $29.1 \%$, respectively. The 6-, 12- and 18-month HCC recurrence rates in patients without prior history of HCC recurrences and in those with prior history of HCC recurrences were 9.2\%, 20.9\%, 24.2\% and 18.5\%, 39.7\%, 39.7\% respectively. Predictive factors for the recurrence of HCC were prior history of HCC and tumor size bigger than $2.5 \mathrm{~cm}$ in diameter ${ }^{[19]}$.

Sixty-eight consecutive cirrhotic patients with HCV and HCC under remission were treated with DAAS ( $n$ $=23$ ) or not treated $(n=45)$. SVR rate reached $96 \%$. Median time between HCC remission and initiation of 
DAAs was 7.2 months, while between the time of starting of DAAs and HCC recurrence was 13.0 months. Recurrence rate was 1.7/100 person-months among treated patients vs. 4.2/100 person-months in not treated patients. HCC recurrence rate was significantly lower in patients who were treated with DAAs compared to untreated group ${ }^{[20]}$.

Mettke et al. ${ }^{[21]}$ compared DAAs treated $(n=158)$, and untreated control patients $(n=184)$ in terms of HCC occurrence. Treated and untreated control patients were followed up for a mean of 440 (91-408) days and a mean of 592 (90-1000) days, respectively. HCC occurred in treated and untreated patients at rates of $2.9 \%$ ( $n$ $=6)$ and $4.48 \%(n=14)$. They concluded that DAAs therapies do not change the short time occurrence of de novo HCC; however, it reduces HCC developing risk after 1.5 years.

In a prospective study of Calvaruso et al. ${ }^{[22]}$, in 2,249 patients with HCV related cirrhosis (Child Pugh A 90.5\%, Child Pugh B 9.5\%) were treated with DAAs. SVR occurred in 95.2\% (2140/2249; Child Pugh A 95.9\%, Child Pugh B 88.3\%; $P<0.001)$. Patients were followed-up for a median of $14(6-24)$ months. In Child Pugh A patients who maintained SVR, HCC developed in $2.1 \%$ of the cases while HCC was seen in $6.6 \%$ of those cases without SVR. Accordingly, in Child Pugh B patients who maintained SVR, HCC developed in $7.8 \%$ of the cases while HCC was observed in $12.4 \%$ of those cases without SVR $(P<0.001)$. The predictive factors for occurrence of HCC were the absence of SVR, serum albumin levels less than $3.5 \mathrm{~g} / \mathrm{dL}$, platelet level $<120 \times 10^{9} / \mathrm{L}$.

Patients with 218 Stage-1 and 226 Stage-2 were treated with PEG-IFN and ribavirin. Patients with SVR had less esophageal varices compared to non-SVR patients (HR 0.23; 95\%CI: 0.11-0.48; $P<0.001$ ). However, there was no difference in terms of the progression of esophageal varices between the groups (HR 458; 95\%CI: 0.33-1.03; $P=0.7)$. SVR was found to be associated with reduced risk of $\mathrm{HCC}^{[23]}$.

In a multicenter retrospective study, 22,500 patients (39\% cirrhotic) were treated with DAAs based regimens. The patients were followed up for 20 months; 19,500 of them had SVR (group A) and 2,982 did not have SVR. A hundred eighty-three patients $(0.9 \%)$ with HCC were detected in Group A, while HCC occurred in 88 patients $(3.4 \%)$ in Group B. (HR 0.28; 95\%CI: 0.22-0.36; $P<0.0001$ ). Even if SVR occurred, the patients over 65 years of age and patients with advanced fibrosis or cirrhosis were associated to increased rates of HCC development. In this study, there were several comorbid conditions like alcohol use (61.4\%), drug addiction (54.2\%), and diabetes mellitus (43.6\%) which all may facilitate HCC development. SVR was associated with $76 \%$ reduced risk of HCC occurrence ${ }^{[24]}$.

In a prospective study, 3,917 patients who included stage F3 fibrosis and CP-A cirrhosis were treated with DAAs based regimen. They were followed up with a mean of $536.2 \pm 197.6$ days after the start of DAAs. Overall incidence of HCC was found to be $0.97 \%$ patients/year, 95\%CI: 0.73-1.26. HCC incidence of cirrhotic patients was found to be $1.18 \%$ patients/year. When patients were stratified according to the stage of liver disease at baseline, HCC incidence rates during the first year of follow-up were 0.46x100 patients/year (95\%CI: 0.12-1.17) in patients with fibrosis F3, 1.49'100 patients/year (95\%CI: 1.03-2.08) in CTP-A cirrhosis and 3.61100 patients/year (95\%CI: 1.86-6.31) in CTP-B cirrhosis. HCC incidence rates in the second year of follow-up declined to $0 \%$ in F3, to $0.20^{\prime} 100$ patients/year (95\%CI: 0.05-0.51) in CTP-A cirrhosis and to $0.69^{\prime} 100$ patients/year (95\%CI: 0.08-2.49) in CTP-B cirrhosis and these differences were statistically significant (Mantel-Cox test, $P=0.00008)^{[25]}$.

In a retrospective study 421 patients who had HCV infection with or without cirrhosis were treated with DAAs therapy. Thirty-three per cent of patients had active or a history of HCC. Twenty-nine out of 421 patients resulted in failed SVR. Twenty-one per cent of patients who had HCC did not have SVR while, SVR failed in $12 \%$ of patients without HCC. Twenty-seven out of 29 patients who failed SVR resulted in active period of HCC. If DAAs treatments were given in an inactive period of HCC or after transplantation, SVR was excellent similar to those without HCC $(P<0.0001)^{[26]}$. 
In a retrospective study 1,170 patients with HCV were treated with DAAs for 12-24 weeks. The patients were followed up for 1.3 years. Twenty-two patients had HCC during the follow-up. Cumulative incidences of HCC were $1.8 \%$ and $2.3 \%$ at 1 year and two years respectively. However, SVR was associated with reduced risk of HCC occurrence in $1.4 \%$ and $1.8 \%$ at 1 year and two years respectively. Non-SVR, hypoalbuminemia, thrombocytopenia, high AFP levels are risk factors for the development of $\mathrm{HCC}^{[27]}$.

The occurrence and recurrence rate of HCC is lower among patients who receive DAAs treatment compared to those who are not treated. $\mathrm{HCV}$ infection should be treated as early as possible in order to reduce the progression of parenchymal damage. Occurrence of SVR after treatment with both DAAs and Interferon is strongly associated with reduced developing or recurrence of HCC in patients with all fibrotic stages and advanced liver diseases compared to those patients who had no SVR. Occurrence of SVR does not exclude the development of HCC. However, reduced recurrence (0.4\%-2\%) of HCC may take a longer time than the patients who had active HCV infection. The patients who had SVR should be followed up periodically in every 3 or 6 months. Presence of active HCC reduces of SVR with the treatment of DAAs. The patients with $\mathrm{HCV}$ infection and active stage of HCC should be treated for HCV infection after curative treatment of HCC and/or after liver transplantation.

In our recently published multi-centric study, 200 patients with chronic hepatitis $\mathrm{C}$ were treated with the fixed dose combination of Sofosbuvir and Ledipasvir for 12 weeks. Thirty-five out of 200 patients had a history of HCC. Nineteen of those 35 patients had curative treatment at the beginning of anti-viral therapy. Median follow-up time was 22.1 months (15.7-30.3 months). Overall HCC occurrence was detected in 18 (9.0\%) out of 200 patients. Recurrence of HCC was detected in 12 out of 16 (75\%) patients who had non-curative treatments, while it was detected in 5 out of $19(26.3 \%)$ patients who had curative treatments. This study also has several limitations. There is no control group, treatment modalities are different, time period between the beginning of anti-viral therapy and the time for recurrence of HCC varies between 3-14 months ${ }^{[28]}$.

Seventy-one million people are still infected with HCV infection in the world. DAAs are very effective with more than $95 \%$ of SVR rates. World Health Organization and some countries like Japan, Egypt, Mongolia, and Turkey have an elimination program for $\mathrm{HCV}$ infection. It may be an important problem to have a claim without evidence that DAAs treatment for HCV infection is associated with the occurrence and recurrence of HCC.

In conclusion, unexpected results concerning high occurrence and recurrence rates of HCC after the treatment of HCV infection with DAAs and complete curative treatment are heterogenic and incompatible in retrospective studies due to clinic, biologic, epidemiologic differences and methodological biases. In most of the studies which are HCV-related, occurrence and recurrence of HCC are retrospective and small case groups. Confounding factors such as age, sex, fibrotic stages, genotypes, Plt, AFP, serum albumine and bilirubine levels, number of liver cirrhosis are different between comparative groups. Curative treatment modalities such as radical surgery and Radio Frequency Ablation, TACE, Trans Arterial Radio Embolization of HCC are different. Prognosis of these modalities are different. Most of the articles did not describe pathological examinations, lymphatic and vascular infiltration of the tumor. Follow-up times are also different in similar studies. In order to make more precise decisions for occurrence or recurrence of HCC after DAAs treatment, we need to do large prospective randomized studies.

\section{DECLARATIONS}

\section{Authors' contributions}

Prof. Necati Örmeci contributed solely to the article.

\section{Availability of data and materials}

Not applicable. 


\section{Financial support and sponsorship}

None.

\section{Conflicts of interest}

Author declared that there are no conflicts of interest.

\section{Ethical approval and consent to participate}

Not applicable.

\section{Consent for publication}

Not applicable.

\section{Copyright}

(C) The Author(s) 2019.

\section{REFERENCES}

1. Conti F, Bionfiglioli F, Scuteri A, Crespi C, Bolondi L, et al. Early occurrence and recurrence of hepatocellular carcinoma in HCVrelated cirrhosis treated with direct-acting antivirals. J Hepatol 2016;65:727-33.

2. Veldt BJ, Heathcote EJ, Wedemeyer H, Reichen J, Hofmann WP, et al. Sustained virologic response and clinical outcomes in patients with chronic hepatitis C and advanced fibrosis. Ann Intern Med 2007;147:677-84.

3. Morgan RL, Baack B, Smith BD, Yartel A, Pitasi M, et al. Eradication of hepatitis C virüs infection and the development of hepatocellular carcinoma: a meta-analysis of observational studies. Ann Intern Med 2013;158:329-37.

4. El-Seraq HB., Kanwal F., Richardson P., Kramer J. Risk of hepatocellular carcinoma after sustained virological response in Veterans with hepatitis C virus infection. Hepatology 2016;64:130-7.

5. Nault JC, Colombo M. Hepatocellular carcinoma and direct acting antiviral treatments:Controversy after the revolution. J Hepatol 2016;65:663-5.

6. Nishiguchi S, Kuroki T, Nakatani S, Morimoto H, Takeda T, et al. Randomised trial of effects of interferon- $\alpha$ on incidence of hepatocellular carcinoma in chronic active hepatitis C with cirrhosis. Lancet 1995;346:1051-5.

7. Reig M, Marino Z, Perello C, Inarrairaegui M, Ribeiro A, et al. Unexpected high rate of early tumour recurrence in patients with HCVrelated HCC undergoing interferon-free therapy. J Hepatol 2016;65:719-26.

8. Morgan RL, Baack B, Smith BD, Yartel A, Pitasi M, et al. Eradication of hepatitis C virus infection and the development of hepatocellular carcinoma. Ann Intern Med 2013;158:329-37.

9. Ravi S, Axley P, Jones D, Kodali S, Simpson H, et al. Unusually high rates of hepatocellular carcinoma after treatment with directacting antiviral therapy for hepatitis C related cirrhosis. Gastroenterology 2017;152:911-3.

10. Cardoso H, Vale AM, Rodrigues S, Gonçalves R, Albuquerque A, et al. High incidence of hepatocellular carcinoma following successful interferon-free antiviral therapy for hepatitis C associated cirrhosis. J Hepatol 2016;65:1057-71.

11. Yang JD, Agel BA, Pungpapong S, Gores GJ, Roberts LR. Direct acting antiviral therapy and tumour recurrence after liver transplantation for hepatitis C-associated hepatocellular carcinoma. J Hepatol 2016;65;856-68.

12. Beste LA, Green PK, Berry K, Kogut MJ, Allison S, et al. Effectiveness of hepatitis C antiviral treatment in a USA cohort of veteran patients with hepatocellular carcinoma. J Hepatol 2017;67:32-9.

13. Yoshida H, Shiratori Y, Moriyama M, Arakawa Y, Ide T, et al. Interferon therapy reduces the risk for hepatocellular carcinoma: national surveillance program of cirrhotic and noncirrhotic patients with chronic hepatitis C in Japan. IHIT Study Group. Inhibition of Hepatocarcinogenesis by Interferon Therapy. Ann Intern Med 1999;131:174-81.

14. Moon C, Jung KS, Kim DY, Baatarkhuu O, Park JY, et al. Lower incidence of hepatocelluler carcinoma and cirrhosis in hepatitis C patients with sustained virological response by pegylated interferon and ribavirin. Dig Dis Sci 2015;60:573-81.

15. Innes H, Barclay ST, Hayes PC, Fraser A, Dillon JF, et al. The risk of hepatocellular carcinoma in cirrhotic patients with hepatitis $\mathrm{C}$ and sustained viral response: role of the treatment regimen. J Hepatol 2018;68:646-54.

16. Telep LE, Muramoto D, Osinusi A, Brainard DM, Reddy KR, et al. No increased risk of hepatocellular carcinoma recurrence in patients following interferon-free, direct acting anti-viral treatment for hepatitis $\mathrm{C}$ virus: a cohort study using large-scale administrative medical claims data. J Hepatol 2017;66:S333-542.

17. Li DK, Ren Y, Fierer DS, Rutledge S, Shaikh OS, et al. The short-term incidence of hepatocellular carcinoma is not increased after hepatitis C treatment with Direct-acting antivirals: an ERCHIVES study. Hepatology 2018;67:2244-53.

18. Petta S, Cabibbo G, Barbara M, Attardo S, Bucci L, et al. Hepatocellular carcinoma recurrence in patients with curative resection or ablation: impact of HCV eradication does not depend on the use of interferon. Aliment Pharmacol Ther 2017;45:160-8.

19. Cabibbo G, Petta S, Calvaruso V, Cacciola I, Cannavo MR, et al. Is early recurrence of hepatocellular carcinoma in HCV cirrhotic patients affected by treatment with direct-acting antivirals? A prospective multicentre study. Aliment Pharmacol Ther 2017;46:688-95.

20. Virlogeux V, Pradat P, Hartig-Lavie K, Bailly F, Maynard M, et al. Direct-acting antiviral therapy decreases hepatocellular carcinoma recurrence rate in cirrhotic patients with chronic hepatitis C. Liver International 2017;37:1122-7.

21. Mettke F, Schlevogt B, Deterding K, Wranke A, Smith A, et al. Interferon-free therapy of chronic hepatitis C with direct-acting 
antivirals does not change the short-term risk for de novo hepatocellular carcinoma in patients with liver cirrhosis. Aliment Pharmacol and Ther 2018;47:516-25.

22. Calvaruso V, Cabibbo G, Cacciola I, Petta S, Madonia S, et al. Incidence of hepatocellular carcinoma in patients with HCV-associated cirrhosis treated with direct-acting antiviral agents. Gastroenterology 2018;155:411-21.

23. Marco VD, Calvaruso V, Ferraro D, Bavetta MG, Cabibbo G, et al. Effects of eradicating hepatitis C virus infection in patients with cirrhosis differ with stage of portal hypertension. Gastroenterology 2016;151:130-9.

24. Kanwal F, Kramer J, Asch SM, Chayanupatkul M, Cao Y, et al. Risk of hepatocellular cancer in HCV patients treated with directacting antiviral agents. Gastroenterology 2017;153:996-1005.

25. Romano A, Angeli P, Piovesan S, Noventa F, Anastassopoulos G, et al. Newly diagnosed hepatocellular carcinoma in patients with advanced hepatitis C treated with DAAs: A prospective population study. J Hepatol 2018;69:345-52.

26. Breitenstein S, Dimitroulis D, Petrowsky H, Puhan MA, Müllhaupt B, et al. Systematic review and meta-analysis of interferon after curative treatment of hepatocellular carcinoma in patients with viral hepatitis. Br J Surg 2009;96:975-81.

27. Minami T, Tateishi R, Nakagomi R, Fujiwara N, Sato M, et al. The impact of direct-acting antivirals on early tumor recurrence after radiofrequency ablation in hepatitis C-related hepatocellular carcinoma. J Hepatol 2016;65:1261-73.

28. Idilman R, Demir M, Aladag M, Erol C, Cavus B, et al. Low recurrence rate of Hepatocellular carcinoma following Ledipasvir and Sofosbuvir treatment in a real-world chronic hepatitis C patients cohort. J Viral Hepat 2019; doi: 10.1111/jvh.13075. 